\title{
Exercise and pregnancy in recreational and elite athletes: 2016/2017 evidence summary from the IOC expert group meeting, Lausanne. Part 5. Recommendations for health professionals and active women
}

\author{
Kari Bø, ${ }^{1,2}$ Raul Artal, ${ }^{3}$ Ruben Barakat, ${ }^{4}$ Wendy J Brown ${ }^{5}$ Gregory A L Davies, ${ }^{6}$ \\ Michael Dooley, ${ }^{7}$ Kelly R Evenson, ${ }^{8}$ Lene A H Haakstad, ${ }^{9}$ Bengt Kayser, ${ }^{10}$ \\ Tarja I Kinnunen, ${ }^{11}$ Karin Larsen, ${ }^{12}$ Michelle F Mottola, ${ }^{13}$ Ingrid Nygaard, ${ }^{14}$ \\ Mireille van Poppel, ${ }^{15}$ Britt Stuge, ${ }^{16}$ Karim M Khan ${ }^{17}$
}

For numbered affiliations see end of article.

\section{Correspondence to} Kari Bø, Department of Sport Medicine, Norwegian School of Sport Science, Oslo 0806, Norway; kari.bo@nih.no

Accepted 6 May 2018 Published Online First 12 June 2018
Check for updates

To cite: $B \varnothing \mathrm{K}$, Artal R, Barakat R, et al. Br J Sports Med 2018:52:1080-1085.

\section{BACKGROUND}

This is part 5 in the series of reviews from the IOC expert committee on exercise and pregnancy in recreational and elite athletes. Part 1 focused on the effects of training during pregnancy and on the management of common pregnancy-related complaints that may be experienced by athletes ${ }^{1}$; part 2 addressed maternal and fetal perinatal outcomes $^{2}$; part 3 reviewed the implications of pregnancy and childbirth on return to exercise and on common illnesses and complaints in the postpartum period. ${ }^{3}$ Part 4 provided recommendations for future research based on parts 1 through $3 .^{4}$ In part 5, we summarise our recommendations for exercise during pregnancy and after childbirth in recreational exercisers and elite athletes experiencing healthy pregnancies. Part 5 also serves as a background for healthcare personnel to advise women who wish to stay active at a high level.

Most of the references to existing research in the respective research areas are listed in the previously published parts ${ }^{1-3}$ and are not repeated here. The recommendations are divided into exercise during conception planning, exercise during pregnancy, effect of exercise during pregnancy on birth outcomes and exercise after childbirth. The level of evidence supporting the majority of the recommendations is very low or low-to-moderate. Therefore, advice to elite athletes regarding exercise frequency, duration and intensity that is beyond current guidelines must be individualised with regular close observation of maternal and fetal well-being.

\section{PRECONCEPTION}

The optimal fertile age period for women coincides with peak performance for many athletes, and some athletes may have impaired fertility related to relative energy deficiency in sport (RED-S). In addition to normal preconception advice, we advise that elite athletes who wish to become pregnant, should discuss specific issues, including their age, body weight, body mass index (BMI), body composition (\% body fat), history of menstrual function, any eating disorders, RED-S and family size intention with their medical team. This team should include a physician with expertise in fertility and pregnancy, such as an obstetrician/gynaecologist. Egg reserve can be estimated using serum anti- Müllerian hormone levels or ultrasound antral follicle count. In elite athletes, these tests require interpretation by an experienced clinician due to background hormonal changes.

Since the fertile age of many athletes overlaps with the peak performance period, family planning is challenging. Adequate information about the possible influence of a pregnancy on performance is critical to making an informed decision. Elite athletes with an uncomplicated pregnancy should be reassured that they can continue exercising, although some adjustments in intensity and activity may be required. If athletes are able to continue exercising at a moderate level throughout gestation they can expect their maximal aerobic capacity $\left(\mathrm{VO}_{2 \max }\right)$ after childbirth to be similar to their prepregnancy levels. ${ }^{13}$ It may be possible to plan pregnancy and childbirth between important competitions (eg, Olympic games), and there is evidence that many elite athletes have performed at the same level or better after uncomplicated pregnancy and birth. ${ }^{5}$ At the same time, there is no evidence to suggest that athletes are protected from many of the musculoskeletal complaints that may occur in relation to pregnancy and childbirth. These may influence athletic performance during pregnancy and after childbirth, but are usually treatable. $^{1-3}$

\section{EXERCISE DURING PREGNANCY Medical screening during pregnancy and additional testing and follow-up}

In the absence of maternal or fetal contraindications, exercise prescription in pregnancy includes the same principles and elements used for the general non-pregnant population, with the distinction that pregnancy is associated with significant anatomical, hormonal, metabolic, cardiovascular and pulmonary changes/adaptations. ${ }^{167}$ 
Box 1 Conditions that post a high risk to the fetus and in which aerobic exercise is absolutely contraindicated

- Haemodynamically significant heart disease.

- Intrauterine growth restriction in current pregnancy.

- Poorly controlled hypertension.

- Restrictive lung disease.

- Cervical insufficiency/cerclage.

- Multiple gestation at risk of premature labour.

- Persistent second or third trimester bleeding.

- Placenta previa after 26 weeks gestation.

- Premature labour during the current pregnancy.

- Ruptured membranes.

- Pre-eclampsia/pregnancy-induced hypertension.

- Severe anaemia.

\section{Screening}

For high level exercisers and elite athletes, a thorough clinical evaluation should be conducted to ascertain that there are no medical or obstetrical reasons to either avoid exercise completely, or to modify exercise routines (box 1,2 ). Absolute contraindications, relative contraindications and warning signs specific to elite athlete athletes exercising in pregnancy have been adapted from the American College of Obstetricians and Gynecologists (ACOG) guidelines. ${ }^{6}$ As pregnancy progresses, new conditions may arise and risk stratification may change from no contraindications, to relative contraindications, to absolute contraindications and athletes and their coaches need to respond accordingly. Given, the lack of research available to counsel elite athletes who choose to exercise above moderate levels, particular attention should be given to fetal growth in the second and third trimester.

\section{Contraindications}

The conditions that are considered high risk and are absolute contraindications to aerobic exercise during pregnancy for elite athletes are mentioned in box 1 .

The conditions that are considered moderate risk, and are relative contraindications to exercise during pregnancy are mentioned in box 2 .

Women without contraindications are recommended to exercise regularly during pregnancy while being regularly reassessed. While engaging in exercise during pregnancy, the following may serve as warning signs to stop exercising:

- vaginal bleeding

- regular painful contractions

- amniotic fluid leakage

- dyspnoea prior to exertion

- dizziness/syncope

Box 2 Conditions that post a moderate risk to the fetus and in which aerobic exercise is relatively contraindicated

- History of fetal growth restriction, miscarriage, premature birth or labour.

- Cervical enlargement.

- Unevaluated maternal cardiac arrhythmia.

- Chronic bronchitis or other respiratory disorders.

- Poorly controlled type I diabetes.

- Extreme underweight.

- Orthopaedic limitations.

- Poorly controlled seizure disorder.
- headache

- chest pain

- muscle weakness

- calf pain or swelling.

Physicians, midwives, physiotherapists and coaches looking for resources to evaluate elite pregnant athletes' risk for exercising in pregnancy are referred to the Physical Activity Readiness Medical Examination (ParMed-X) for Pregnancy, a guideline for health screening prior to participation in a prenatal fitness class or other exercise. ${ }^{8}$

\section{Advice on some general concerns related to exercise Hyperthermia}

Raising body core temperature above $103^{\circ} \mathrm{F}\left(39^{\circ} \mathrm{C}\right)$ due to infection, in the first days of pregnancy may increase the risk of fetal neural tube defect abnormalities. The risk is highest 20-30 days after conception. Hyperthermia should be avoided especially during the first trimester. Exercise at $60 \%-70 \%$ of $\mathrm{VO}_{2 \max }$ does not raise core temperature above $38^{\circ} \mathrm{C}$. Moderate exercise during gestation will not raise body core temperature above this threshold. Thermoregulation steadily improves during pregnancy, as reflected by a gradual decline in rectal temperature. ${ }^{9-11}$

\section{Supine position}

There have been few studies evaluating the maternal and fetal effect of exercising in the supine position, although caution is recommended in many guidelines due to the potential risk of decreased venous return and decreased uterine blood flow. Orthostatic hypotension may occur from supine position and standing motionless. Should side effects, such as dizziness, occur while engaging in such activities, they should be discontinued. Side effects are more likely to occur after 28 weeks gestation. To reduce potential risks, exercises normally done in the supine position may be modified by tilting the torso to 45 degrees or doing the exercises in side-lying, sitting or standing positions. Some studies report no adverse effect with exercise in the supine position for 2-3 min at a time. ${ }^{12} 13$

\section{Exercise at high altitude}

A special case concerns low-land athletes who want to train at high altitude (eg, cross-country skiers). Since both hypoxia and exercise decrease blood flow to the uterus, and their combination leads to a decrease in arterial oxygen saturation, it seems advisable to refrain from high-intensity training regimens at altitudes $>1500-2000 \mathrm{~m}$ during pregnancy for those not acclimatised.

\section{Nutrition}

Pregnant women are advised to follow their country-specific recommendations and seek advice from their antenatal care providers regarding nutrient supplementation before and during pregnancy. Pregnant elite athletes should also pay special attention to adequate energy intake to achieve recommended gestational weight gain. ${ }^{14}$

High-intensity endurance training and testing of $\mathrm{VO}_{2 \max }$

Like recommended for pregnant women in the general population, pregnant elite athletes may use perception of exertion or fatigue to gauge their training intensity. Pregnant elite athletes who have access to more sophisticated evaluation are recommended to refrain from training at intensities $>90 \%$ of their $\mathrm{VO}_{2 \max }$, although there has not yet been research in pregnant athletes whose baseline $\mathrm{VO}_{2 \max }$ is already significantly higher than the general population (eg, cross-country skiers). 


\section{Strength training}

There is little scientific evidence on the risks of resistance training during pregnancy. In general it is advised that athletes pay attention to technique and safety. Caution is recommended when weight lifting on the back due to the concerns related to vena cava compression as discussed under 'Supine position'. If there is a feeling of muscle strain or excessive fatigue, exercises should be modified to avoid injury. Strenuous strength training should be adjusted to avoid the Valsalva manoeuvre and excessive pressure towards the pelvic floor. The pelvic floor muscles should be contracted before and during heavy lifting to counteract the impact on the pelvic floor from increased intra-abdominal pressure.

There is strong evidence that strength training of the pelvic floor muscles during pregnancy can prevent and treat urinary incontinence during pregnancy and after birth. ${ }^{16}$ Near maximal pelvic floor contractions, in sets of $8-12$, performed three times per day on most days is recommended. Pelvic floor muscle training during pregnancy has been shown to reduce the length of both the first and second stage of labour without negative influence on childbirth. ${ }^{17}$

\section{Sports to be avoided}

High-risk sport can be divided into those with risk of falling, of direct trauma to the abdominal wall and physiologic risk.

\section{Scuba diving}

Scuba diving is a popular activity. Pregnant women should refrain from scuba diving, because the fetus is not protected from decompression problems and is at risk of malformation and gas embolism after decompression disease. ${ }^{18}$

\section{Direct trauma and risk of falling}

Full contact Olympic sports such as wrestling, boxing, judo, taekwondo, rugby and ice hockey should be avoided throughout gestation, although non-contact training may be continued.

The following Olympic sports should also be avoided, especially during second and third trimester: football (soccer), handball, basketball, equestrian, field hockey, trampoline, gymnastics artistic, diving, BMX and mountain cycling, volleyball, some track and field events, downhill and freestyle skiing, ski jumping, luge, bobsleigh, skeleton, snowboard, short track speed skating and some figure skating events where there is the possibility for contact with other athletes or the environment and subsequent damage to the fetus/placenta. Non-contact training may be continued.

\section{Advice for common complaints and diseases during pregnancy \\ Nausea}

The nausea, vomiting and fatigue that is common in early pregnancy may lead to underperformance during training and competition. Early drug treatment for nausea and vomiting may be required and should be done in association with the appropriate medical authorities and governing bodies (see https:// www.wada-ama.org/sites/default/files/prohibited_list_2018_en. pdf).

\section{Fatigue}

Pregnancy should be considered in the differential diagnosis of the 'tired female athlete/exercise fatigue'. Elite athletes should plan for more time for sleep and rest during pregnancy.
Mental health and well-being

In addition to the general aspects of being pregnant and becoming a mother, athletes may be particularly concerned about training intensity, stress during competition and whether they will be able to return to the same level of performance and regain their position on a team after childbirth. This may need special focus and follow-up.

\section{Gestational weight gain}

Due to limited research in pregnant elite athletes, the first line indicator of sufficient energy intake for the growth and development of the fetus should be adequate gestational weight gain as suggested by the Institute of Medicine. ${ }^{14}$ More frequent ultrasound assessment should be used when clinically indicated, usually in the context of persistently lower than expected symphysis-fundal height growth.

\section{Gestational diabetes}

It is likely that pregnant recreational and elite athletes are at lower risk of gestational diabetes than the general population. However, for those that do develop gestational diabetes and require insulin therapy, the pregnant athlete and her coach should be aware that more intense training days or competition may require a reduction in insulin dose.

\section{Pre-eclampsia}

The association between physical activity and risk of pre-eclampsia remains unclear in the general population and particularly among athletes where evidence is lacking. ${ }^{19-25}$ Pre-eclampsia is a severe condition in pregnancy and athletes with this condition must be followed up adequately by their pregnancy care provider.

\section{Gestational hypertension}

If present in elite athletes, close follow-up is needed as for other pregnant women.

\section{Oedema}

For women with significant leg oedema in pregnancy, hydrotherapy may complement other treatments such as compression stockings and leg elevation.

\section{Musculoskeletal complaints \\ Pelvic floor dysfunction}

Many sports include high forces on the pelvic floor. Pregnant athletes are not at lower risk than others of experiencing urinary or faecal incontinence or pelvic organ prolapse. There is strong evidence that pelvic floor muscle training during pregnancy can prevent and treat urinary incontinence. ${ }^{16}$ Pregnant athletes are therefore advised to learn how to contract the pelvic floor muscles correctly before giving birth, and to incorporate specific strength training of the pelvic floor muscles into their daily training regimen for prevention. If symptoms are present, assessment and training by a gynaecologist/women's health physiotherapist is indicated.

\section{Low back and pelvic girdle pain}

Elite athletes are not at lower risk of low back or pelvic girdle pain in pregnancy, and special attention should be paid to screening for these conditions and referral to sport/women's health physiotherapist for proper individual management. 


\section{Diastasis recti abdominis}

There is no evidence of effective prevention or treatment options for this condition during pregnancy.

\section{EXERCISE AND BIRTH OUTCOMES Miscarriage}

Light-intensity to moderate-intensity leisure-time physical activity does not increase the risk of miscarriage and may perhaps decrease it. ${ }^{26}$ One study found an increased risk of miscarriage after higher than average physical strain on days 6-9 after the estimated date of ovulation (relative risk (RR) 2.5, 95\% CI 1.3 to 4.6$).{ }^{27}$ One large epidemiological study reported an increased risk of miscarriage in women who performed high impact exercise $\geq 7$ hours per week during the first trimester. ${ }^{28}$ However, there is insufficient data to guide the pregnant elite athlete about miscarriage risk when they participate in high-intensity, speed, endurance or weight training in the first trimester.

\section{Preterm birth}

There is a lack of evidence on the risk of preterm birth in elite athletes. Some, ${ }^{29-31}$ but not all, ${ }^{32}$ reviews on workplace data have demonstrated an increase in risk with excessive standing and heavy lifting at work that should be considered when advising the pregnant athlete.

\section{Induction, episiotomy, epidural}

There is insufficient evidence to draw conclusions about the effects of habitual strenuous physical activity on the rate of labour induction, episiotomy or epidural analgesia.

\section{Prolonged labour}

There is some evidence to suggest that the first stage of labour is shortened in exercising pregnant women. There is insufficient evidence to determine whether the musculoskeletal adaptations observed in elite athletes predispose them to a longer or shorter second stage of labour. As mentioned above, pelvic floor muscle training shortens both the first and second stage of labour. ${ }^{17}$

\section{Caesarean birth}

There is insufficient evidence regarding the risk of caesarean birth in the elite athlete. However, elite athletes are more likely to have a normal BMI, which is associated with a decreased risk of caesarean birth. In the general population, two meta-analyses indicated reduced risk of caesarean birth among those participating in interventions, which usually included aerobic activity, compared with control conditions, ${ }^{21} 33$ while other meta-analyses did not show a reduction in risk. ${ }^{23} 34$

\section{Risk of levator ani muscle defect and anal sphincter tear}

There is insufficient evidence regarding the risk of levator ani muscle defect and anal sphincter tear in the elite athlete. It is unlikely that an elite athlete's risk for these birth complications differs from that of the general population.

\section{Newborn birth weight}

In the general population, three meta-analyses concluded that babies of more active women usually weigh less than babies of less active women, ranging from 1 to $60 \mathrm{~g}$ in difference ${ }^{20234}$ (da Silva 2017 from cohort studies only). However, other meta-analyses did not find these differences in birth weight ${ }^{2021253536}$ (da Silva 2017 for randomised controlled trial only), and there are no studies in elite athletes.

\section{Apgar score}

The Apgar score is not affected by the level of maternal physical activity. $^{253336}$

\section{EXERCISE IN THE POSTPARTUM PERIOD Return to sports}

Pregnancy and childbirth impacts the maternal musculoskeletal system, and vaginal birth can be likened to the impact of an acute sport injury. The 2016 Consensus statement on return to sport from the First World Congress in Sports Physical Therapy concluded that deciding when to return to sport after injury is complex and multifactorial, and that research evidence to support return to sport decisions is scant. ${ }^{37}$ The authors explained return to sport as a continuum comprising three elements, which may also serve as a model for return to sport after childbirth:

- Return to participation: participating in rehabilitation, training or in sport, but at a lower level than before pregnancy.

- Return to sport: return to her defined sport, but not performing at her previous level. Some athletes may be satisfied with this level after childbirth.

- Return to performance: the athlete has gradually returned to her defined sport and is performing at or above her prepregnancy level.

\section{Some general advice \\ Endurance training}

Women who have been regularly exercising at least at a moderate level during pregnancy, can expect their $\mathrm{VO}_{2 \max }$ to return to their prepregnancy level or even higher after delivery. In the postpartum period, endurance training should start gradually. Low impact activities such as cross-country skiing, fast walking, low impact aerobics and step training put little pressure on the pelvic floor and can start soon after birth.

\section{Strength training}

Strength training in the postpartum period should start gradually with a 'pelvic floor muscle first' focus. This can be done with a pre-contraction of the pelvic floor muscle and holding the contraction during exercises that are accompanied by increased intra-abdominal pressure (eg, bench press, heavy abdominal exercise, leg press and squat). Focus should also be on abdominal and back muscles.

\section{Breast feeding}

$\mathrm{WHO}^{38}$ recommends exclusive breast feeding for at least 6 months. Exercise during the period of breast feeding is safe and helps women resume normal weight. Elite athletes who exercise intensively may lose too much weight and should compensate with higher energy intake. Athletes may find exercise more comfortable after breast feeding. Breastfeeding athletes may also find a fitted bra with features of greater breast elevation more comfortable than a standard encapsulation sport bra.

\section{Advice for common postpartum complaints and diseases Musculoskeletal complaints Pelvic floor}

Given the need for a strong pelvic floor in most sports, strength training of the pelvic floor muscles can start directly after birth. In particular, if injuries to the peripheral nerves, fascia/ligaments or the pelvic floor muscles have occurred, focused rehabilitation maybe indicated. Exercisers with symptoms of urinary or faecal incontinence or a feeling of a bulge in the vagina should be 
referred to a gynaecologist for assessment and supervised intensive strength training/physiotherapy of the pelvic floor.

\section{Low back and pelvic girdle pain}

One small study found that the prevalence of low back and pelvic girdle pain was the same in athletes and controls. ${ }^{39}$ The lumbopelvic region in elite athletes undergoes substantial loading and stress in many sports, with a potential for increased risk of low back and pelvic girdle pain. There is limited knowledge on prevention and treatment. Athletes presenting with low back and pelvic girdle pain at 6 weeks post partum should be referred to a sport/women's health physiotherapist.

\section{Diastasis recti abdominis}

It is likely that elite athletes have the same prevalence of diastasis recti abdominis as the general population. Most sports require strong abdominal muscles. To date, there is no evidence to guide elite athletes on which abdominal exercises are the most effective in reducing the distance between the two rectus muscles and strengthening the abdominals post partum. If the condition persists post partum and is affecting the athlete's performance, referral for physiotherapy and/or surgical correction is indicated.

\section{Postpartum depression}

There is a limited, but growing, body of evidence to suggest that physical activity before, during or after pregnancy may reduce the risk of postpartum depression. ${ }^{40-42}$ Very little is known about postpartum depression in athletes. Elite athletes may need extra support and help with both the typical postpartum stressors in addition to the stress associated with training for performance at an international level.

\section{Postpartum weight loss}

Athletes resuming training soon after delivery can expect to return to their prepregnancy weight within 6 months of childbirth. To avoid excess weight loss, athletes who are breast feeding while training should ensure adequate dietary intake to support their energy and nutritional needs.

\section{Pelvic floor pain}

It is likely that athletes are affected by the same risk factors for development of pelvic floor pain as non-athletes. Pelvic pain can be located in the pelvic floor muscles, but given that many factors contribute to pelvic pain, a careful evaluation is needed to attribute such pain to the pelvic floor. Long-term pelvic floor muscle pain has deleterious effects on quality of life, sexual function and other important parameters.

\section{Sexual dysfunction}

Sexual dysfunction is common after childbirth and generally improves over the course of the first postpartum year and in particular after stopping breast feeding with a return to normal oestrogen levels. Based on biologic plausibility, it is unlikely that this condition affects athletes differently from non-athletes.

\section{CONCLUSION}

Increasing numbers of elite female athletes reach their peak performance and want to continue competing during the years of optimal fertility. The evidence summarised and the recommendations from the 2016 IOC expert group meeting in Lausanne revealed a significant lack of high-quality evidence specific to pregnant elite athletes and especially the impact of high-intensity exercise and extreme doses of exercise during pregnancy on maternal and newborn outcomes, as well as an athlete's ability to return to their competitive peak after childbirth. The advice and recommendations given here are therefore based on scientific evidence when available, and on consensus between 16 experts representing different countries, healthcare systems and disciplines.

Given the infrequency of pregnancy in athletes competing at the national or international level in any given time period, and the geographic challenges of enrolling these women from around the globe in prospective research trials, we recommend that the IOC develop a prospective international registry of elite competitive athletes who plan to become, or are pregnant to allow for the collection and analysis of maternal and neonatal consequences of extreme levels of exercise before, during and after gestation. Such a registry would provide the IOC with relevant data that can be used to guide athletes and sport federations in optimising maternal and child health, ultimately enhancing the female athlete's performance.

\section{Author affiliations}

${ }^{1}$ Sports Medicine, Norwegian School of Sport Sciences, Oslo, Norway

2Department of Obstetrics and Gynecology, Akershus University Hospital, Lørenskog, Norway

${ }^{3}$ Department of Obstetrics/Gynecology and Women's Health, St Louis University, St Louis, Missouri, USA

${ }^{4}$ Facultad de Ciencias de la ActividadFísica y del Deporte—INEF, Universidad Politécnica de Madrid, Madrid, Spain

${ }^{5}$ Centre for Research on Exercise Physical Activity and Health, School of Human Movement and Nutrition Sciences, University of Queensland, St Lucia, Queensland, Australia

${ }^{6}$ Queens University, Kingston, Ontario, Canada

${ }^{7}$ The Poundbury Clinic Dorchester-The Poundbury Suite, King Edward VII Hospital, London, UK

${ }^{8}$ USA Gillings School of Global Public Health, Department of Epidemiology, University of North Carolina, Chapel Hill, North Carolina, USA

${ }^{9}$ Department of Sport Sciences, Norwegian School of Sport Sciences, Oslo, Norway

${ }^{10}$ Faculty of Biology and Medicine, Institute of Sport Science, University of Lausanne, Lausanne, Switzerland

${ }^{11}$ Faculty of Social Sciences/Health Sciences, University of Tampere, Tampere, Finland

${ }^{12}$ The Swedish School of Sport and Health Sciences, Stockholm and Department of Community Medicine and Rehabilitation/Sports Medicine Unit, Umeå University, Umeå, Sweden

${ }^{13}$ R. Samuel McLaughlin Foundation-Exercise and Pregnancy Lab, The University of Western Ontario, London, Ontario, Canada

${ }^{14}$ Department of Obstetrics and Gynecology, University of Utah, Utah, Salt Lake City, USA

${ }^{15}$ Institute of Sport Science, University of Graz, Graz, Austria

${ }^{16}$ Division of Orthopaedic Surgery, Oslo University Hospital, Oslo, Norway

${ }^{17}$ Department of Family Practice \& School of Kinesiology, Centre for Hip Health and

Mobility, University of British Columbia, Vancouver, British Columbia, Canada

Funding The authors have not declared a specific grant for this research from any funding agency in the public, commercial or not-for-profit sectors.

Competing interests None declared.

Patient consent Not required.

Provenance and peer review Not commissioned; internally peer reviewed.

(c) Article author(s) (or their employer(s) unless otherwise stated in the text of the article) 2018. All rights reserved. No commercial use is permitted unless otherwise expressly granted.

\section{REFERENCES}

1 Bø K, Artal R, Barakat $R$, et al. Exercise and pregnancy in recreational and elite athletes: 2016 evidence summary from the IOC expert group meeting, Lausanne. Part 1 -exercise in women planning pregnancy and those who are pregnant. Br J Sports Med 2016;50:571-89.

2 Bø K, Artal R, Barakat R, et al. Exercise and pregnancy in recreational and elite athletes: 2016 evidence summary from the IOC expert group meeting, Lausanne. Part 2-the effect of exercise on the fetus, labour and birth. Br J Sports Med 2016;50:1297-305.

$3 \mathrm{Bø} \mathrm{K}$, Artal R, Barakat R, et al. Exercise and pregnancy in recreational and elite athletes: 2016/17 evidence summary from the IOC Expert Group Meeting, Lausanne. Part 3-exercise in the postpartum period. Br J Sports Med 2017;51:1516-25. 
$4 \mathrm{Bø} \mathrm{K}$, Artal R, Barakat R, et al. Exercise and pregnancy in recreational and elite athletes: 2016/17 evidence summary from the IOC expert group meeting, Lausanne. Part 4-Recommendations for future research. Br J Sports Med 2017;51:1724-6.

5 Erdener U, Budgett R. Exercise and pregnancy: focus on advice for the competitive and elite athlete. Br J Sports Med 2016;50:567.

6 Committee opinion no. 650 summary: physical activity and exercise during pregnancy and the postpartum period. Obstet Gynecol 2015;126:1326-7.

7 Davies GA, Wolfe LA, Mottola MF, et al. Exercise in pregnancy and the postpartum period. J ObstetGynaecol Can 2003;25:516-29.

8 Canadian Society for Exercise Physiology. PARMed-X for pregnancy physical activity readiness medical examination. $2015 \mathrm{http}: / / \mathrm{www}$. csep.ca/cmfiles/publications/parq/ parmed-xpreg.pdf

9 Lindqvist PG, Marsal K, Merlo J, et al. Thermal response to submaximal exercise before, during and after pregnancy: a longitudinal study. J Matern Fetal Neonatal Med 2003;13:152-6

10 Brearley AL, Sherburn M, Galea MP, et al. Pregnant women maintain body temperatures within safe limits during moderate-intensity aqua-aerobic classes conducted in pools heated up to 33 degrees Celsius: an observational study. J Physiother 2015:61:199-203.

$110^{\prime}$ Neill ME. Maternal rectal temperature and fetal heart rate responses to upright cycling in late pregnancy. Br I Sports Med 1996;30:32-5.

12 Barakat R, Pelaez M, Cordero Y, et al. Exercise during pregnancy protects against hypertension and macrosomia: randomized clinical trial. Am J Obstet Gynecol 2016:214:649.e1-649.e8.

13 Barakat R, Perales $M$, Cordero $Y$, et al. Influence of land or water exercise in pregnancy on outcomes: a cross-sectional study. Med Sci Sports Exerc 2017;49:1397-403.

14 IOM. (Institute of Medicine) and NRC (National Research Council). Weight gain during pregnancy: reexamining the guidelines. Washington DC: The National Academies Press, 2009.

15 Maughan RJ, Shirreffs SM. IOC Consensus Conference on Nutrition in Sport, 25-27 October 2010, International Olympic Committee, Lausanne, Switzerland. J Sports Sci 2011;29 Suppl 1:S1.

16 Boyle R, Hay-Smith EJ, Cody JD, et al. Pelvic floor muscle training for prevention and treatment of urinary and faecal incontinence in antenatal and postnatal women. Cochrane Database Syst Rev 2012;10:CD007471.

17 Du Y, Xu L, Ding L, et al. The effect of antenatal pelvic floor muscle training on labor and delivery outcomes: a systematic review with meta-analysis. Int Urogynecol J 2015;26:1415-27.

18 Reid RL, Lorenzo M. SCUBA Diving in Pregnancy. J Obstet Gynaecol Can 2018. doi: 10.1016/j.jogc.2017.11.024. [Epub ahead of print].

19 Aune D, Saugstad OD, Henriksen T, et al. Physical activity and the risk of preeclampsia: a systematic review and meta-analysis. Epidemiology 2014;25:331-43.

20 da Silva SG, Ricardo LI, Evenson KR, et al. Leisure-time physical activity in pregnancy and maternal-child health: a systematic review and meta-analysis of randomized controlled trials and cohort studies. Sports Med 2017;47:295-317.

21 Di Mascio D, Magro-Malosso ER, Saccone G, et al. Exercise during pregnancy in normal-weight women and risk of preterm birth: a systematic review and metaanalysis of randomized controlled trials. Am J Obstet Gynecol 2016;215:561-71.

22 Kasawara KT, do Nascimento SL, Costa ML, et al. Exercise and physical activity in the prevention of pre-eclampsia: systematic review. Acta Obstet Gynecol Scand 2012;91:1147-57.
23 Muktabhant B, Lawrie TA, Lumbiganon P, et al. or both, for preventing excessive weight gain in pregnancy. Cochrane Database Syst Rev 2015;6:CD007145.

24 Wolf HT, Owe KM, Juhl M, et al. Leisure time physical activity and the risk of preeclampsia: a systematic review. Matern Child Health J 2014;18:899-910.

25 Zheng J, Wang H, Ren M. Influence of exercise intervention on gestational diabetes mellitus: a systematic review and meta-analysis. J Endocrinol Invest 2017;40:1027-33.

26 Schlüssel MM, Souza EB, Reichenheim ME, et al. Physical activity during pregnancy and maternal-child health outcomes: a systematic literature review. Cad Saude Publica 2008;24(Suppl 4):s531-s544.

27 Hjollund NH, Jensen TK, Bonde JP, et al. Spontaneous abortion and physical strain around implantation: a follow-up study of first-pregnancy planners. Epidemiology 2000:11:18-23.

28 Madsen $\mathrm{M}$, Jørgensen $\mathrm{T}$, Jensen $\mathrm{ML}$, et al. Leisure time physical exercise during pregnancy and the risk of miscarriage: a study within the Danish National Birth Cohort. BJOG 2007;114:1419-26.

29 Bonzini M, Coggon D, Palmer KT, et al. Risk of prematurity, low birthweight and pre-eclampsia in relation to working hours and physical activities: a systematic review. Occup Environ Med 2007;64:228-43.

30 Palmer KT, Bonzini M, Bonde JP, et al. Pregnancy: occupational aspects of management: concise guidance. Clin Med 2013;13:75-9.

31 van Beukering MD, van Melick MJ, Mol BW, et al. Physically demanding work and preterm delivery: a systematic review and meta-analysis. Int Arch Occup Environ Health 2014:87:809-34.

32 Savitz DA, Murnane P. Behavioral influences on preterm birth: a review. Epidemiology 2010;21:291-9.

33 Wiebe HW, Boulé NG, Chari R, et al. The effect of supervised prenatal exercise on fetal growth: a meta-analysis. Obstet Gynecol 2015;125:1185-94.

34 Thangaratinam S, Rogozinska E, Jolly K, et al. Effects of interventions in pregnancy on maternal weight and obstetric outcomes: meta-analysis of randomised evidence. BMJ 2012;344:e2088.

35 Han S, Middleton P, Crowther CA. Exercise for pregnant women for preventing gestational diabetes mellitus. Cochrane Database Syst Rev 2012;7:CD009021.

36 Yu Y, Xie R, Shen $C$, et al. Effect of exercise during pregnancy to prevent gestational diabetes mellitus: a systematic review and meta-analysis. J Matern Fetal Neonatal Med 2018;31:1632-7.

37 Ardern CL, Glasgow P, Schneiders A, et al. 2016 Consensus statement on return to sport from the First World Congress in Sports Physical Therapy, Bern. Br I Sports Med 2016:50:853-64

38 World Health Organization (WHO). Recommendations on exclusive breast feeding, 2017.

39 Bo K, Backe-Hansen KL. Do elite athletes experience low back, pelvic girdle and pelvic floor complaints during and after pregnancy? Scand I Med Sci Sports 2007:17:480-7.

40 Teychenne M, York R. Physical activity, sedentary behavior, and postnatal depressive symptoms: a review. Am J Prev Med 2013;45:217-27.

41 McCurdy AP, Boulé NG, Sivak A, et al. Effects of exercise on mild-to-moderate depressive symptoms in the postpartum period: a meta-analysis. Obstet Gynecol 2017;129:1087-97.

42 Poyatos-León R, García-Hermoso A, Sanabria-Martínez G, et al. Effects of exercisebased interventions on postpartum depression: A meta-analysis of randomized controlled trials. Birth 2017;44:200-8. 